ESAIM: PROCEEDINGS AND SURVEYS, 2020, Vol. 68, p. 35-51

Hervé Cardot \& Pierre Calka

\title{
RANDOM WALKS IN RANDOM SCENERIES AND RELATED MODELS *
}

\author{
FRANÇOISE PÈnE ${ }^{1}$
}

\begin{abstract}
We present random walks in random sceneries as well as three related models: $U$-statistics indexed by random walks, a model of stratified media with inhomogeneous layers (random one-way streets) and the one-dimensional Lévy-Lorentz gas (random roundabouts on a line). We present in particular results obtained in collaboration with Castell, Guillotin-Plantard, Br. Schapira, Franke, Wendler, Aurzada, Bianchi and Lenci.

Résumé. Nous présentons le modèle des promenades aléatoires en paysages aléatoires ainsi que trois autres modèles connexes: $U$-statistiques indexées par une marche aléatoire, un modèle de marche aléatoire sur un réseau stratifié avec orientation aléatoire des strates (sens de circulation aléatoires) et finalement le modèle du gaz de Lévy-Lorentz unidimensionnel (ronds-points aléatoires sur une droite). Nous présentons notamment des résultats obtenus en collaboration avec Castell, Guillotin-Plantard, Br. Schapira, Franke, Wendler, Aurzada, Bianchi et Lenci.
\end{abstract}

\section{INTRODUCTION}

The aim of this paper is to show that random walks in random sceneries are processes of multiple interests. They are both interesting on their own, and related to other natural models

At the end of the 1970's, random walks in random sceneries (RWRS) have been introduced and studied as a probabilistic model by Borodin [8,9] and Kesten and Spitzer [27], ten years later by Bolthausen [7] for a two-dimensional version, and more recently by many authors. This probabilistic model is a famous example of model with long time dependence, which is stationary but non Markov under the annealed law, and Markov but not stationary under the quenched law, and which (in some cases) converges after normalization to a self similar process with stationary increments, which is neither a Lévy process, nor a fractional Brownian motion.

Random walks in random sceneries are also linked with ergodic theory. Indeed, when the random walk is the simple symmetric random walk on $\mathbb{Z}$, the random walk in random scenery corresponds to an ergodic sum of a dynamical system, the so-called $T, T^{-1}$-transformation. This dynamical system has been introduced in a list of open problems by Weiss [36, problem 2, p. 682] in the early 1970's. It is a famous natural example of $K$-transformation which is not Bernoulli and even not loosely Bernoulli as this has been shown by Kalikow in $[26]$.

In the present paper, we focus on probabilistic limit theorems of random walks in random sceneries and of three related models, for which we point out their link with random walk in random sceneries.

In Section 1, we introduce random walks in random sceneries, and present a selection of limit theorems in this context such as distributional convergence, local limit theorem, recurrence/transience. In particular, we

* ANR project MALIN, ANR-16-CE93-0003, IUF

${ }^{1}$ Université de Brest, LMBA, UMR CNRS 6205, IUF

(C) EDP Sciences, SMAI 2020

This is an Open Access article distributed under the terms of the Creative Commons Attribution License (http://creativecommons.org/licenses/by/4.0), which permits unrestricted use, distribution, and reproduction in any medium, provided the original work is properly cited. 
will discuss the different normalizations, the different limits (either a Lévy process or a stochastic integral with respect to a Lévy process) as well as the different convergence topologies.

In Section 2, we present an extension of random walks in random sceneries to bivariate observables $h(x, y)$. The corresponding process is called $U$-statistic indexed by a random walk. We will see that different behaviours can occur in this context, with a dichotomy in the case of observables in $L^{4}$, for which either the behaviour is as if the observable was a sum $g(x)+g(y)$, or as if it was a product $\phi_{x} \phi_{y}$ and in any of these two cases the $U$-statistics can be approximated by an expression involving a RWRS. But, when the observables are in the normal domain of attraction of a $\beta$-stable distribution with $\beta<2$ (which is the natural context to consider when the observable is not square integrable), a totally different behaviour can occur with a limit similar to those of RWRS but involving a Lévy sheet instead of a Lévy process.

In Section 3, we present a model introduced by Matheron and de Marsily in [31] as a simple model for the displacement of a pollutant in an inhomogeneous stratified media. It is a model of random walk in $\mathbb{Z}^{2}$ with random orientations of the vertical lines. The transience of this model has been proved by Campanino and Petritis in [11]. We recall this result as well as a limit theorem established by Guillotin-Plantard and Le Ny in [22], a local limit theorem established by Castell, Guillotin-Plantard, Schapira and the author in [13] and also estimates on persistence probability established by Aurzada, Guillotin-Plantard and the author in [1]. A crucial point in the study of this model is that the second coordinate of the process can be approximated by a RWRS. In this section, we also mention an open model: the random Manhattan model with random orientations of both vertical and horizontal lines, for which very few has been proved at the present time.

Finally, in Section 4, we consider the one-dimensional Lévy-Lorentz gas which has been introduced by Barkai, Fleurov and Klafter in [2] as a one-dimensional toy model for transport in porous media. In this model, a particle goes straight on the real line at unit speed and can only change its direction (with probability $1 / 2$ ) when it reaches some positions (called roundabouts in the present paper), these positions having been randomly fixed at the beginning, with independent and identically distributed gaps between two consecutive roundabouts. Whereas a quenched central limit theorem (with the standard normalization) has been established by Bianchi, Cristadoro, Lenci and Ligabò in [4] when the gaps between the roundabouts are integrable, a very different behaviour appears when the gaps are not integrable but in the normal domain of attraction of a $\beta$-stable distribution with $0<\beta<1$. In this non-integrable setting, a result of convergence in distribution to a non càdlàg process has been proved by Bianchi, Lenci and the author in [5]. A key point is that this process can be mathematically described with the use of a RWRS.

\section{RANDOM WALKS IN RANDOM SCENERIES (RWRS)}

\subsection{Definitions and assumptions}

To define a random walk in random scenery, we need two ingredients: a random walk $S$ and a random scenery $\zeta$.

Random walk on $\mathbb{Z}^{d}$. Let us consider a random walker moving on $\mathbb{Z}^{d}$, starting from 0 and making independent and identically distributed steps $X_{1}, X_{2}, \ldots$ The position of the walker at time $n$ is given by $S_{n}:=\sum_{k=1}^{n} X_{k}$.

Random scenery. To each site $\ell$ of $\mathbb{Z}^{d}$, we associate a real valued random variable $\zeta_{\ell}$. These random variables are assumed to be independent and identically distributed.

Independence assumption. The random walk $S=\left(S_{n}\right)_{n}$ and the random scenery $\zeta=\left(\zeta_{\ell}\right)_{\ell \in \mathbb{Z}^{d}}$ are assumed to be independent one from the other.

Random walk in random scenery. We associate an amount to the walker in the following manner. We assume that the walker has the amount 0 at the begining and that he wins the amount $\zeta_{\ell}$ each time he visits 
the site $\ell \in \mathbb{Z}^{d}$. Thus, at time $n$, the total amount won by the walker is

$$
Z_{n}:=\sum_{k=1}^{n} \zeta_{S_{k}}
$$

Because of our independence assumptions, it will be worthwhile to notice that $Z_{n}$ can be rewritten in the following manner

$$
Z_{n}=\sum_{x \in \mathbb{Z}^{d}} \zeta_{x} N_{n}(x)
$$

with $N_{n}(x):=\#\left\{k=1, . ., n: S_{k}=x\right\}$ the number of visits of the walk $S$ to the site $x \in \mathbb{Z}^{d}$ up to time $n$ $\left(\left(N_{n}(x)\right)_{n \geq 0, x \in \mathbb{Z}^{d}}\right.$ is the local time of $\left.S\right)$.

Properties. On the first hand, the process $Z=\left(Z_{n}\right)_{n \geq 0}$ has stationary increments but is not Markov. On the other hand, conditionally to $\zeta, Z$ is Markov but its increments are not stationary. So, either in quenched or in annealed setting, we cannot use classical arguments made for Markov processes with stationary increments. We will actually, in some cases, exhibit a behaviour far from the classical behaviour of Markov processes with stationary increments.

Additional Assumptions on $S$ and $\zeta$.

On the random walk $S$, we consider two cases:

(a) either $S$ is transient,

(b) or there exists $\alpha \in[d, 2]$ such that the distribution of $X_{1}$ is in the normal domain of attraction of an $\alpha$-stable distribution, i.e. $\left(n^{-\frac{1}{\alpha}} S_{n}\right)_{n}$ converges in distribution to an $\alpha$-stable random wariable $\mathcal{W}_{1}$. This assumption is equivalent to the fact that there exists an $\alpha$-stable càdlàg process $\mathcal{W}:=\left(\mathcal{W}_{t}\right)_{t \geq 0}$ such that

$$
\forall T>0, \quad\left(n^{-\frac{1}{\alpha}} S_{\lfloor n t\rfloor}\right)_{t \in[0, T]} \stackrel{\mathcal{L}, J_{1}}{\longrightarrow}\left(\mathcal{W}_{t}\right)_{t \in[0, T]} \quad \text { as } n \rightarrow+\infty
$$

where $\stackrel{\mathcal{L}, J_{1}}{\longrightarrow}$ means the convergence in distribution with respect to the $J_{1}$-metric (see Subsection 1.2 .3 for recalls on this metric).

Observe that Case (b) with $\alpha<d$ is contained in Case (a).

Concerning the scenery $\zeta$, we assume that there exists $\beta \in(0,2]$ such that the distribution of $\zeta_{1}$ is in the normal domain of attraction of a strictly $\beta$-stable distribution, i.e. $\left(n^{-\frac{1}{\beta}} \sum_{k=1}^{n} \zeta_{k}\right)_{n}$ (identifying $k$ with $\left.(k, \ldots, k) \in \mathbb{Z}^{d}\right)$ converges in distribution to a strictly $\beta$-stable random variable $Y_{1}$, with characteristic function $\phi$ given by

$$
\phi(u)=e^{-|u|^{\beta}\left(A_{1}+i A_{2} \operatorname{sgn}(u)\right)} \quad u \in \mathbb{R},
$$

where $0<A_{1}<\infty$ and $\left|A_{1}^{-1} A_{2}\right| \leq|\tan (\pi \beta / 2)|$. When $\beta=1$, we will further assume the symmetry condition $\sup _{t>0} \mathbb{E}\left[\zeta_{0} \mathbf{1}_{\left\{\zeta_{0} \leq t\right\}}\right]<+\infty$. These assumptions on $\zeta$ imply the existence of two independent identically distributed $\beta$-stable càdlàg processes $Y^{+}:=\left(Y_{x}^{+}\right)_{x>0}$ and $Y^{-}:=\left(Y_{x}^{-}\right)_{x>0}$ such that

$$
\forall T>0, \quad\left(n^{-\frac{1}{\beta}} \sum_{k=1}^{\lfloor n x\rfloor} \zeta_{k}, n^{-\frac{1}{\beta}} \sum_{k=1}^{\lfloor n y\rfloor} \zeta_{-k}\right)_{x, y \in[0, T]} \stackrel{\mathcal{L}, J_{1}}{\longrightarrow}\left(Y_{x}^{+}, Y_{y}^{-}\right)_{x, y \in[0, T]} \quad \text { as } n \rightarrow+\infty
$$

\subsection{Limit theorems for RWRS}

\subsubsection{Results}

Borodin studied the case $\alpha=\beta=2, d=1$ in [8] and the case when $S$ is transient and $\beta=2$. Approximately at the same time, Kesten and Spitzer studied in [27] the more general case $d=1<\alpha \leq 2$ and some aspects of the case when $S$ is transient. Then Bolthausen studied the case $\alpha=d=\beta=2$ in [7]. Deligiannidis-Utev studied 
the case $\alpha=d=1, \beta=2$ in [17], bringing some complements to [7]. Ben Arous and Cerný and Fontes and Mathieu, motivated by trap models, studied in $[3,19]$ the case $S$ transient, $\beta<1$ and $\zeta_{x} \geq 0$. Finally Castell, Guillotin-Plantard and the author studied in [12] the case $\beta<2$ when $S$ is transient or $\alpha=d \in\{1,2\}$.

The results of convergence in distribution are summarized in the following table in which $\stackrel{\mathcal{L}, \text { f.d.d. }}{\longrightarrow}$ means the convergence of the finite dimensional distributions, $\stackrel{\mathcal{L}, J_{1}}{\longrightarrow}$ and $\stackrel{\mathcal{L}, M_{1}}{\longrightarrow}$ mean the convergence in distribution in the space of càdlàg functions endowed with respectively the $J_{1}$ and the $M_{1}$ metric (see Subsection 1.2.3 for some recalls and comparisons of these metrics).

\begin{tabular}{|c|c|}
\hline Assumption on $S$ & Limit theorem \\
\hline transient &  \\
\hline$\alpha=d \in\{1,2\}$ & 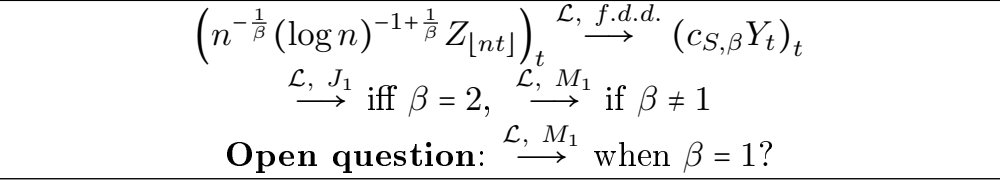 \\
\hline$d=1<\alpha \leq 2$ & $\begin{array}{l}\left(n^{-1+\frac{1}{\alpha}-\frac{1}{\alpha \beta}} Z_{\lfloor n t\rfloor}\right)_{t} \stackrel{\mathcal{L}, J_{1}}{\longrightarrow}\left(\Delta_{t}=\int_{0}^{+\infty} L_{t}(x) d Y_{x}^{+}+\int_{0}^{+\infty} L_{t}(-x) d Y_{x}^{-}\right)_{t} \\
\text { where } L \text { is the local time of } \mathcal{W}: \int_{[0, t]} f\left(\mathcal{W}_{t}\right) d t=\int_{\mathbb{R}} f(x) L_{t}(x) d x\end{array}$ \\
\hline
\end{tabular}

Comments. When the walk $S$ is transient, $Z_{n}$ behaves roughly as in the easiest case when $S_{n}=(n, \ldots, n)$ in which $Z_{n}=\sum_{k=1}^{n} \zeta_{k}$.

In the critical case when $\alpha=d$, then the limit is the same as when $S$ is transient, with a slight change of normalization.

In these two cases, the limit $Y$ is a stable process, which is càdlàg and which is continuous if and only if $\beta=2$. The behaviour is much different when $d=1<\alpha \leq 2$. In this case, the limit is an analogous of $Z$ in continuous time. Indeed, $\Delta$ is obtained from $Z_{n}=\sum_{x \in \mathbb{Z}^{d}} \zeta_{x} N_{n}(x)$ by replacing $\sum_{x} \zeta_{x} \ldots$ by $\int_{\mathbb{R}} \ldots d Y_{x}$ and by replacing the local time $N_{n}(x)$ of $S$ by the local time $L_{t}(x)$ of $\mathcal{W}$ (both replacements corresponding to some convergence). The limit $\Delta$ is a continuous self-similar process with stationary but not independent increments, it is not a stable process.

About the constant $c_{S, \beta}$. If $S$ is transient, then $c_{S, \beta}=\mathbb{E}\left[N_{\infty}^{\beta-1}\right]^{1 / \beta}$ where $N_{\infty}=\sum_{k \in \mathbb{Z}} \mathbf{1}_{\left\{S_{k}=0\right\}}$, up to complete $S$ in a two-sided random walk $\left(S_{n}\right)_{n \in \mathbb{Z}}\left(\left(S_{-n}\right)_{n \geq 0}\right.$ being an independent copie of $\left.\left(-S_{n}\right)_{n}\right)$.

If $\alpha=d \in\{1,2\}$, then $c_{S, \beta}=\left(\frac{\Gamma(\beta+1)}{(\pi A)^{\beta-1}}\right)^{\frac{1}{\beta}}$, with

- $A=2 \sqrt{\operatorname{det} \Sigma^{2}}$ if $\alpha=d=2$ and if $\Sigma^{2}$ is the variances-covariances matrix of $S_{1}$,

- $A$ is the positive real number such that the characteristic function of $\mathcal{W}_{1}$ is $\mathbb{E}\left[e^{i t \mathcal{W}_{1}}\right]=e^{-A|t|}$ for every $t \in \mathbb{R}$.

\subsubsection{Heuristic explanations}

Heuristic explanations about the normalizations. The different normalizations can be, very heuristically, explained by the following rough computation, that can be made rigorous. Let us consider the range $\mathcal{R}_{n}$ of $S$ up to time $n$, that is the number of different sites visited by $S$ up to time $n: \mathcal{R}_{n}=\#\left\{S_{1}, \ldots, S_{n}\right\}=\#\{x \in$ $\left.\mathbb{Z}^{d}: N_{n}(x)>0\right\}$. Now, considering roughly that for most of the visited $x$ (i.e. most of the $x \in \mathbb{Z}^{d}$ such that $\left.N_{n}(x)>0\right), N_{n}(x) \approx \frac{n}{\mathcal{R}_{n}}$, we can make the following very heuristic computation

$$
Z_{n}=\sum_{x \in \mathbb{Z}^{d}} \zeta_{x} N_{n}(x)=\sum_{x \in \mathbb{Z}^{d}: N_{n}(x)>0} \zeta_{x} N_{n}(x) \approx \sum_{k=1}^{\mathcal{R}_{n}} \zeta_{k} \frac{n}{\mathcal{R}_{n}} \approx\left(\mathcal{R}_{n}\right)^{\frac{1}{\beta}} \frac{n}{\mathcal{R}_{n}}=n \mathcal{R}_{n}^{\frac{1}{\beta}-1}
$$


Now the order of magnitude of $\mathcal{R}_{n}$ is $n$ if $S$ if transient, $\frac{n}{\log n}$ if $\alpha=d$ and $n^{\frac{1}{\alpha}}$ if $d=1<\alpha \leq 2$ (see for example [35, p. 26], [28, Theorem 6.9 p.698] and [28, Equation (7.a) p.703]).

Ideas of the proofs of convergence. Let us explain the simple ideas behind the technical proofs. As usual the proofs of these results rely on the proof of the convergence of the finite dimensional distributions

$$
\left(\nu_{n}^{-1} Z_{\left\lfloor n t_{1}\right\rfloor}, \ldots, \nu_{n}^{-1} Z_{\left\lfloor n t_{m}\right\rfloor}\right)_{n}, \quad t_{1}, \ldots, t_{m}>0, m \in \mathbb{N}^{*}
$$

(where $\nu_{n}$ is the suitable normalization) and on a tightness argument. Whereas the tightness argument depends strongly on the assumptions on $S, \zeta$ (for example we used a result by Louhichi and Rio in [30] to prove the convergence in $M_{1}$ when $\alpha=d$ and $\beta>1$ ), the proof of the finite dimensional distributions relies on the same idea (with some variations) for all the above mentioned limit results. Let us explicit this idea in the simplest case when $m=1$ and $t_{1}=1$ and when $\mathbb{E}\left[e^{i x \zeta_{0}}\right] \sim e^{-c_{0}|x|^{\beta}}$ as $x \rightarrow 0$, the extension to the general situation being just technical. To prove the convergence in distribution, we use characteristic functions. The rough idea is to prove that, for every $t \in \mathbb{R}$,

$$
\begin{aligned}
\mathbb{E}\left[e^{i t \nu_{n}^{-1} Z_{n}}\right] & =\mathbb{E}\left[\mathbb{E}\left[e^{i t \nu_{n}^{-1} Z_{n}} \mid S\right]\right]=\mathbb{E}\left[\mathbb{E}\left[e^{i t \nu_{n}^{-1} \sum_{x \in \mathbb{Z}^{d}} \zeta_{x} N_{n}(x)} \mid S\right]\right] \\
& =\mathbb{E}\left[\prod_{x \in \mathbb{Z}^{d}} \mathbb{E}\left[e^{i t \nu_{n}^{-1} N_{n}(x) \zeta_{0}} \mid S\right]\right] \\
& \sim \mathbb{E}\left[\prod_{x \in \mathbb{Z}^{d}} e^{-c_{0} \nu_{n}^{-\beta}\left|t N_{n}(x)\right|^{\beta}}\right]=\mathbb{E}\left[e^{-c_{0}|t|^{\beta} \nu_{n}^{-\beta} \sum_{x \in \mathbb{Z}^{d}}\left|N_{n}(x)\right|^{\beta}}\right],
\end{aligned}
$$

where we used successively the fact that, knowing $S$, the $\zeta_{x}$ are i.i.d. and the form of their common characteristic function, the conclusion then follows from a result of convergence in distribution of $\nu_{n}^{-\beta} \sum_{x}\left|N_{n}(x)\right|^{\beta}$ (for example, in the case $\alpha=d$ and $\beta<2$, we used results obtained by Cerný in [15]).

\subsubsection{Modes of convergence}

Every convergence in the above tabular is valid in the sense of the finite dimensional distribution (f.d.d.) and stronger in most of the cases.

When the limit is continuous (which happens when $\beta=2$ or when $\alpha>d=1$ ), the convergence holds for the classical $J_{1}$ metric. But in the other cases $(\beta<2$ and $S$ transient or $\alpha=d)$, the above mentioned distributional limit theorems have been established in a weaker sense:

- for the $M_{1}$ metric if $\beta \neq 1$ (and if $S$ is transient or $\alpha=d$ ),

- only in the sense of finite dimensional distribution if $\beta=1$ (and if $S$ is transient or $\alpha=d$ ).

Even worse, it has been proved in [12] that the sequence of processes is not tight for the $J_{1}$ metric if $\beta<2$ and $\alpha=d$.

To understand this point, let us define roughly the $J_{1}$ and $M_{1}$ metrics on the set of càdlàg functions as follows (see $[6,37])$.

Given two càdlàg functions $f$ and $g$ defined on $[0, T], d_{J_{1}}(f, g)\left(\right.$ resp. $d_{M_{1}}(f, g)$ ) is the infimum of the $\ell$ such that two "ants" can travel simultaneously one the graph of $f$ and the other the graph of $g$, staying at a $|\cdot|_{\infty}$-distance less than or equal to $\ell$ one from the other, jumping (resp. walking vertically) when they meet a discontinuity, and without return.

The following pictures illustrates different situations. 


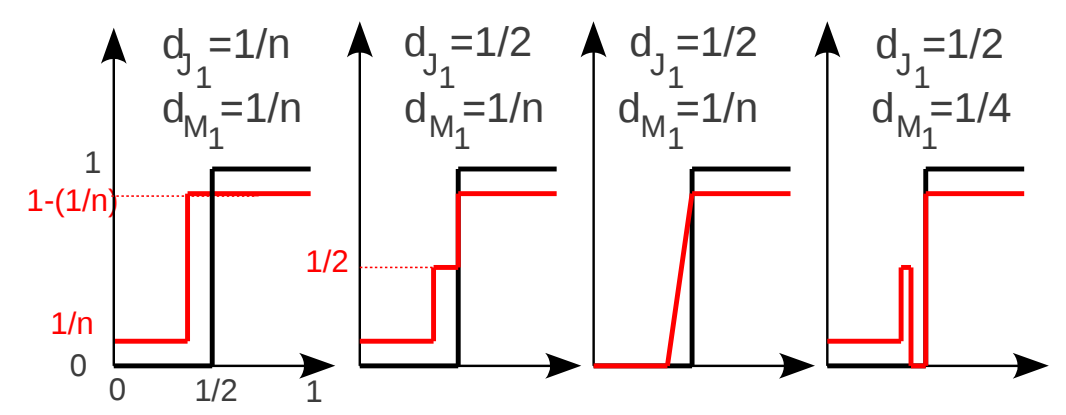

These pictures illustrate in particular the important fact that a sequence of continuous functions cannot converge to a discontinuous one in the $J_{1}$ metric, whereas this becomes possible for the $M_{1}$-metric. We used this fact to prove the non-tightnes for $J_{1}$ when $\beta<2$ and $\alpha=d$.

\subsection{Local limit theorems, recurrence, transience}

The above mentioned limit theorems state that the sequence of processes $\left(\left(\nu_{n}^{-1} Z_{\lfloor n t\rfloor}\right)_{t}\right)_{n}$ converges in distribution, as $n \rightarrow+\infty$ to some limit process $\left(\widetilde{\mathcal{Z}}_{t}\right)_{t}$ with

(a) $\nu_{n}:=n^{\frac{1}{\beta}}$ if $S$ is transient,

(b) $\nu_{n}:=n^{\frac{1}{\beta}}(\log n)^{1-\frac{1}{\beta}}$ if $\alpha=d \in\{1,2\}$,

(c) $\nu_{n}:=n^{1-\frac{1}{\alpha}+\frac{1}{\alpha \beta}}$ if $\alpha>d=1$.

\subsubsection{Local limit theorems}

Local limit theorems have been stated by Castell, Guillotin-Plantard, Br. Schapira and the author in [12,13] with different statements in the lattice and in the nonlattice cases.

Let us consider for example the simplest case when $\zeta_{0}$ takes integer values and has a non-arithmetic distribution (i.e. $\mathbb{Z}$ is the additive group generated by $\left.\left\{a-b ; \mathbb{P}\left(\zeta_{1}=a\right) \mathbb{P}\left(\zeta_{1}=b\right)>0\right\}\right)$. Then estimates on $\mathbb{P}\left(Z_{n}=\left\lfloor\nu_{n} x\right\rfloor\right)$ have been established in $[12,13]$ and in particular we proved that

$$
\mathbb{P}\left(Z_{n}=0\right) \sim f_{1}(0) \nu_{n}^{-1},
$$

with $f_{1}$ the density function of $\widetilde{\mathcal{Z}}_{1}$. Let us indicate that a multi-time local limit theorem has also been established in [14] in the case $\alpha=\beta=2$ implying that

$$
\exists c_{2}>1, \quad \mathbb{P}\left(Z_{n}=Z_{2 n}=0\right) \sim c_{2}\left[\mathbb{P}\left(Z_{n}=0\right)\right]^{2} \text {, i.e. } \mathbb{P}\left(Z_{2 n}-Z_{n}=0 \mid Z_{n}=0\right) \sim c_{2} \mathbb{P}\left(Z_{2 n}-Z_{n}=0\right) .
$$

This exhibits a behaviour very different from the case of random walks for which

$$
\mathbb{P}\left(S_{n}=S_{2 n}=0\right)=\left[\mathbb{P}\left(S_{n}=0\right)\right]^{2} .
$$

This shows the long memory of the process $Z$.

\subsubsection{Recurrence, transience}

As a direct consequence of the above local limit theorem, we get that

$$
\beta<1 \Rightarrow Z \text { is transient. }
$$

Indeed $\beta<1 \Rightarrow \mathbb{E}\left[\sum_{n \geq 0} \mathbf{1}_{\left\{Z_{n}=0\right\}}\right]=\sum_{n \geq 0} \mathbb{P}\left(Z_{n}=0\right)<\infty$, and so, a.s., $Z$ returns to 0 only a finite number of times (ensuring the transience). 
Moreover, we made the remark that the converse is true. Indeed, results of ergodic theory established by Schmidt in [33] and in [34, Theorem 11] ensure that

$$
\beta \geq 1 \Rightarrow Z \text { is recurrent, }
$$

where recurrence of $Z$ means that, a.s., $Z$ returns infinitely often to 0 (this remark comes from [13] when $\beta>1$ and from an informal remark by Guillotin-Plantard when $\beta=1$ ).

\section{EXTENSIONS OF RWRS: U-STATISTICS INDEXED BY A RANDOM WALK}

Given a measurable space $(E, \mathcal{E})$, we consider a random walk $S=\left(S_{n}\right)_{n}$ on $\mathbb{Z}^{d}$ as in the previous section and a sequence $\left(\xi_{x}\right)_{x \in \mathbb{Z}^{d}}$ of $E$-valued independent identically distributed random variables, independent of $S$. We are interested in sums of observables of $\left(\xi_{S_{k}}\right)_{k=1, \ldots, n}$. The case where the observables are $h\left(\xi_{S_{k}}\right)$ corresponds to RWRS and has been considered in the previous section. In this section, we investigate the case of bivariate observables $h\left(\xi_{S_{j}}, \xi_{S_{k}}\right)$, with $h: E \times E \rightarrow \mathbb{R}$ measurable. This leads us to the study of the following quantities

$$
U_{n}:=\sum_{j, k=1}^{n} h\left(\xi_{S_{j}}, \xi_{S_{k}}\right)
$$

which we call $U$-statistic indexed by a random walk. Up to replacing $h(x, y)$ by $(h(x, y)+h(y, x)) / 2$, we assume without any loss of generality that $h$ is a symmetric function. We assume moreover that we can neglect the diagonal terms $h\left(\xi_{S_{j}}, \xi_{S_{j}}\right)$ in $U_{n}$ (in practice this is true if, for example, $h(x, x)=0$ or, in the cases we will consider, if $h\left(\xi_{x}, \xi_{x}\right)$ has the same distribution as $h\left(\xi_{x}, \xi_{y}\right)$ for $\left.x \neq y\right)$.

$U$-statistics indexed by a random walk provide an extension of random walk in random scenery and also of $U$-statistics $\sum_{1 \leq j<k \leq n} h\left(\xi_{j}, \xi_{k}\right)$ (which corresponds to $U$-statistics indexed by the transient random walk $S_{k}=k$ ). $U$-statistics have been studied by many authors. Let us mention namely [25], [24], [16].

\subsection{A dichotomy for observables admitting a moment of order 4}

Guillotin-Plantard and her coauthors studied the case when $\mathbb{E}\left[\left(h\left(\xi_{0}, \xi_{1}\right)\right)^{4}\right]<\infty$ and $\mathbb{E}\left[h\left(\xi_{0}, \xi_{1}\right)\right]=0$. They exhibit the fact that, in this situation, the behaviour of $U_{n}$ depends on $\mathbb{E}\left[h\left(\xi_{0}, \xi_{1}\right) \mid \xi_{1}\right]=g\left(\xi_{1}\right)$, with $g(x):=$ $\mathbb{E}\left[h\left(\xi_{0}, x\right)\right]$.

Cabus and Guillotin-Plantard studied in [10] the cases when $\alpha=d=2$ or $S$ transient. Guillotin-Plantard and Ladret studied in [23] the case when $d=1 \leq \alpha \leq 2$. The results in these contexts can be separated in two cases depending on $g\left(\xi_{1}\right)$.

Roughly speaking, only two behaviours can occur: either $U_{n}$ behaves as if $h$ was a $\operatorname{sum} h(x, y)=g(x)+g(y)$ (case $(\Sigma)$ below) or $U_{n}$ behaves as if $h(x, y)$ was a product $h(x, y)=\phi_{x} \phi_{y}$ (or more precisely as if $U_{n}$ was an "infinite linear combination" of such processes) (case (П) below).

\subsubsection{Nondegenerate case}

( $\Sigma)$ If $\mathbb{E}\left[\left(g\left(\xi_{1}\right)\right)^{2}\right]>0$, then

$$
U_{n} \approx \sum_{j, k=1}^{n}\left(g\left(\xi_{S_{j}}\right)+g\left(\xi_{S_{k}}\right)\right)=2 n \sum_{k=1}^{n} g\left(\xi_{S_{k}}\right)=n \sum_{k=1}^{n} 2 \zeta_{S_{k}}, \quad \text { with } \quad \zeta_{x}:=g\left(\xi_{x}\right) \text {. }
$$

In this case, $U_{n}$ behaves as if $h(x, y)$ was a sum $g(x)+g(y)$ and so $U_{n}$ behaves as $n$ times the RWRS $Z_{n}=$ $\sum_{k=1}^{n} 2 g\left(\xi_{S_{k}}\right)$ with $\beta=2$. Since

$$
\left(\left(\nu_{n}\right)^{-1} Z_{\lfloor n t\rfloor}\right)_{t} \stackrel{\mathcal{L}, J_{1}}{\longrightarrow}\left(\widetilde{\mathcal{Z}}_{t}\right)_{t},
$$


this leads to

$$
\left(\left(n \nu_{n}\right)^{-1} U_{\lfloor n t\rfloor}\right)_{t} \stackrel{\mathcal{L}, J_{1}}{\longrightarrow}\left(\widetilde{\mathcal{Z}}_{t}\right)_{t}
$$

Observe that the normalization of $U_{n}$ is $n \nu_{n}$, where $\nu_{n}$ is the normalization of the RWRS $Z$.

\subsubsection{Degenerate case}

(П) If $g\left(\xi_{1}\right)=0$ a.s., then $h(x, y)=\sum_{i} \lambda_{i} \phi_{x}^{(i)} \phi_{y}^{(i)}$, with $\mathbb{E}\left[\phi_{\xi_{x}}^{(i)}\right]=0, \mathbb{E}\left[\phi_{\xi_{x}}^{(i)} \phi_{\xi_{x}}^{(j)}\right]=\delta_{i, j}$ and

$$
U_{n} \approx \sum_{i} \lambda_{i} \sum_{j, k=1}^{n} \phi_{\xi_{S_{j}}}^{(i)} \phi_{\xi_{S_{k}}}^{(i)}=\sum_{i} \lambda_{i}\left(\sum_{k=1}^{n} \zeta_{S_{k}}^{(i)}\right)^{2}, \quad \text { with } \quad \zeta_{x}^{(i)}:=\phi_{\xi_{x}}^{(i)}
$$

This means that, in this case, $U_{n}$ behaves roughly as if $h(x, y)$ was a product $\phi_{x} \phi_{y}$ and, more precisely, that $U_{n}$ behaves as $\sum_{i} \lambda_{i}\left(Z_{n}^{(i)}\right)^{2}$ with $Z^{(i)}$ the RWRS given by $Z_{n}^{(i)}:=\sum_{k=1}^{n} \zeta_{S_{k}}^{(i)}$ with $\beta=2$. Since

$$
\left(\left(\nu_{n}\right)^{-1} Z_{\lfloor n t\rfloor}^{(i)}\right)_{i, t} \stackrel{\mathcal{L}, J_{1}}{\longrightarrow}\left(\widetilde{\mathcal{Z}}_{t}^{(i)}\right)_{i, t}
$$

this leads to

$$
\left(\left(\nu_{n}\right)^{-2} U_{\lfloor n t\rfloor}\right)_{t} \stackrel{\mathcal{L}, J_{1}}{\longrightarrow}\left(\sum_{i} \lambda_{i}\left(\widetilde{\mathcal{Z}}_{t}^{(i)}\right)^{2}\right)_{t} .
$$

In particular the normalization of $U_{n}$ is $\left(\nu_{n}\right)^{2}$, where $\nu_{n}$ is the normalization of the RWRS $Z_{n}^{(i)}$.

\subsection{Results for non square integrable observables}

Franke, Wendler and the author investigated situations in which $\mathbb{E}\left[\left(h\left(\xi_{0}, \xi_{1}\right)\right)^{2}\right]=\infty$. In this case, we made the following natural assumptions (natural extension of the square integrable case):

- either $g(x):=\mathbb{E}\left[h\left(\xi_{0}, x\right)\right]$ is well defined and $g\left(\xi_{1}\right)$ is in the normal domain of attraction of a strictly $\beta$-stable distribution,

- or the distribution of $h\left(\xi_{1}, \xi_{2}\right)$ is in the normal domain of attraction of a strictly $\beta$-stable distribution.

\subsubsection{Nondegenerate case}

We first studied in [20] the following generalization of the case $(\Sigma)$ :

$(\Sigma *)$ If there exist $1<\beta<\beta^{\prime}$ such that $\mathbb{E}\left[\left|h\left(\xi_{1}, \xi_{2}\right)\right|^{\frac{2 \beta^{\prime}}{1+\beta^{\prime}}}\right]<\infty$ and such that the distribution of $\zeta_{k}:=g\left(\xi_{k}\right)$ (with $g(x):=\mathbb{E}\left[h\left(\xi_{0}, x\right)\right]$ ) is in the normal domain of attraction of a strictly $\beta$-stable random variable, then

$$
U_{n} \approx \sum_{j, k=1}^{n}\left(\zeta_{S_{j}}+\zeta_{S_{k}}\right)=2 n \sum_{k=1}^{n} \zeta_{S_{k}}
$$

Observe that, since $\frac{2 \beta}{1+\beta}<\beta$, in $(\Sigma *)$, the integrability assumption on $h\left(\xi_{1}, \xi_{2}\right)$ is weaker than the integrability assumption on its conditional expectation $\zeta_{1}$.

As in the previous case $(\Sigma)$, we proved that

$$
\left(\left(n \nu_{n}\right)^{-1} U_{\lfloor n t\rfloor}\right)_{t} \stackrel{\mathcal{L}}{\longrightarrow}\left(\widetilde{\mathcal{Z}}_{t}\right)_{t}
$$

with $\nu_{n}$ and $\widetilde{\mathcal{Z}}$ given by

$$
\left(\left(\nu_{n}\right)^{-1} Z_{\lfloor n t\rfloor}\right)_{t} \stackrel{\mathcal{L}}{\longrightarrow}\left(\widetilde{\mathcal{Z}}_{t}\right)_{t}, \quad \text { with } Z \text { the RWRS } \quad Z_{n}=\sum_{k=1}^{n} 2 g\left(\xi_{S_{k}}\right)
$$


Idea of the proof of $(\Sigma *)$. As for $(\Sigma)$, the idea is to use the Hoeffding decomposition (see [25]) of $U_{n}$ given by

$$
U_{n}=2(n-1) \sum_{i=1}^{n} g\left(\xi_{S_{i}}\right)+R_{n}, \quad \text { with } R_{n}:=\sum_{i, j=1, \ldots, n, i \neq j}\left[h\left(\xi_{S_{i}}, \xi_{S_{j}}\right)-g\left(\xi_{S_{i}}\right)-g\left(\xi_{S_{i}}\right)\right],
$$

and to prove that the contribution of $R_{n}$ can be neglected.

\subsubsection{Convergence to an integral with respect to a Lévy sheet}

We exhibited in [21] general assumptions under which the behaviour of $U_{n}$ is neither similar to $(\Sigma)$ nor to (I), but to a new behaviour $(\Psi)$ described below.

We won't detail here our technical assumptions. Let us just give an intuition of them. Our most technical assumption means that $h\left(\xi_{x}, \xi_{y}\right)$ and $h\left(\xi_{x}, \xi_{z}\right)$ behave, in some weak sense, as independent random variables as soon as $y \neq z$.

To fix idea, let us indicate that an example satisfying our general technical assumptions is given by

$h(x, y)=\mathbf{1}_{|x| \neq|y|}\left(\mathbf{1}_{\{x y>0\}}-\mathbf{1}_{\{x y<0\}}\right)|| x|-| y \|^{-\frac{1}{\beta}}$, with $\xi_{0} \mathbb{R}$ - valued, admitting an even and bounded density.

( $\Psi)$ If $h\left(\xi_{0}, \xi_{1}\right)$ is in the normal domain of attraction of a strictly $\beta$-stable distribution $($ with $0<\beta<2$ ) and under additional technical assumptions, then

$$
U_{n} \approx 2^{1-\frac{1}{\beta}} \widetilde{U}_{n}, \quad \text { with } \widetilde{U}_{n}:=\sum_{j, k=1}^{n} \zeta_{S_{j}, S_{k}}=\sum_{x, y \in \mathbb{Z}^{d}} \zeta_{x, y} N_{n}(x) N_{n}(y)
$$

where $\left(\zeta_{x, y}\right)_{x, y \in \mathbb{Z}^{d}}$ are independent random variables with the same distribution as $h\left(\xi_{0}, \xi_{1}\right)$ and where $\approx$ means here an approximation in distribution.

Let us explain this approximation very roughly. The rough idea is that our assumptions which are close to the independence of the $\left(h\left(\xi_{x}, \xi_{z}\right)\right)_{z \in \mathbb{Z}^{d}}$ imply that

$$
U_{n}=2 \sum_{x<y} h\left(\xi_{x}, \xi_{y}\right) N_{n}(x) N_{n}(y) \approx 2 \sum_{x<y} \zeta_{x, y} N_{n}(x) N_{n}(y)
$$

with $<$ a total strict order on $\mathbb{Z}^{d}$, but

$$
\widetilde{U}_{n}=\sum_{x<y}\left(\zeta_{x, y}+\zeta_{y, x}\right) N_{n}(x) N_{n}(y) \approx 2^{\frac{1}{\beta}} \sum_{x<y} \zeta_{x, y} N_{n}(x) N_{n}(y)
$$

since the random variables $\zeta_{x, y}$ are in the normal domain of attraction of a $\beta$-stable transformation (in particular, if the common distribution of the $\zeta_{x, y}$ was the limit stable distribution, then the last above $\approx$ would be an equality in distribution). This leads to $U_{n} \approx 2^{1-\frac{1}{\beta}} \widetilde{U}_{n}$.

Let us state our results. We consider the same process $\mathcal{W}$ and the same notation $c_{S, \beta}$ as for RWRS $\left(\mathcal{W}\right.$ was given in $(1), c_{S, \beta}$ appears in the first tabular and is defined just after it). We consider moreover four independent identically distributed Lévy sheets $\mathcal{Y}^{( \pm, \pm)}$such that

$$
\left(\sum_{k=1}^{\lfloor n t\rfloor} \sum_{\ell=1}^{\lfloor n s\rfloor} \zeta_{\epsilon k, \epsilon^{\prime} \ell}\right)_{s, t>0} \stackrel{\mathcal{L}}{\longrightarrow}\left(\mathcal{Y}_{s, t}^{\left(\epsilon, \epsilon^{\prime}\right)}\right)_{s, t}, \quad \forall \epsilon, \epsilon^{\prime} \in\{-,+\}
$$

Under our general technical assumptions, we have the following convergence results. 


\begin{tabular}{|c|c|}
\hline Assumption on $S$ & Limit theorem \\
\hline transient & $\begin{array}{c}\left(n^{-\frac{2}{\beta}} U_{\lfloor n t\rfloor}\right)_{t} \stackrel{\mathcal{L}, \stackrel{f . d . d .}{\longrightarrow}\left(2^{1-\frac{1}{\beta}} c_{S, \beta}^{2} \mathcal{Y}_{t, t}^{(+,+)}\right)_{t}}{\longrightarrow} \stackrel{\mathcal{L}, M_{1}}{\longrightarrow} \text { if } \beta<1 \\
\text { Open question: } \stackrel{\mathcal{L}, J_{1}}{\longrightarrow} \text { when } \beta<2 ?\end{array}$ \\
\hline$\alpha=d \in\{1,2\}$ &  \\
\hline$d=1<\alpha \leq 2$ & $\begin{array}{c}\left(n^{-2+\frac{2}{\alpha}-\frac{2}{\alpha \beta}} Z_{\lfloor n t\rfloor}\right)_{t} \stackrel{\mathcal{L}, J_{1}}{\longrightarrow}\left(2^{1-\frac{1}{\beta}} \sum_{\epsilon, \epsilon^{\prime} \in\{+,-\}} \int_{[0,+\infty)^{2}} L_{t}(\epsilon x) L_{t}\left(\epsilon^{\prime} y\right) d \mathcal{Y}_{x, y}^{\left(\epsilon, \epsilon^{\prime}\right)}\right)_{t} \\
\text { where } L \text { is the local time of } \mathcal{W}(\text { defined in }(1))\end{array}$ \\
\hline
\end{tabular}

Observe that, whereas the normalization is analogous to the one of ( $\Pi)$ in the sense that it is the square of the normalization $\nu_{n}$ of a RWRS with the same $\beta$ and the same $S$, the limit is not the square of the limit of the RWRS but it is, in some sense, an extension of the limit process of this RWRS obtained by replacing the $\beta$-stable process $Y$ by the $\beta$-stable Lévy sheet $\mathcal{Y}$ and $L_{t}(x)$ by $L_{t}(x) L_{t}(y)$.

Idea of the proof of $(\Psi)$. As in [16] in the easiest context of $U$-statistics, our proof uses point processes. The proof of the convergence of $\left(U_{n}\right)_{n}$ relies on the fact that

$$
\nu_{n}^{-2} U_{n}=\int_{\mathbb{R}} x d \mathcal{N}_{n}(x), \quad \text { with } \mathcal{N}_{n}:=\sum_{x, y \in \mathbb{Z}^{d}} \delta_{\nu_{n}^{-2}} h\left(\xi_{x}, \xi_{y}\right) N_{n}(x) N_{n}(y)
$$

combined with the convergence in distribution (conditionnally to $S$ ) of the sequence of point processes $\left(\mathcal{N}_{n} / 2\right)_{n}$ to a Poisson point process, with $\nu_{n}:=n^{\frac{1}{\beta}}$ if $S$ is transient, with $\nu_{n}:=n^{\frac{1}{\beta}}(\log n)^{1-\frac{1}{\beta}}$ if $\alpha=d$ and with $\nu_{n}:=n^{1-\frac{1}{\alpha}+\frac{1}{\alpha \beta}}$ if $d=1<\alpha \leq 2$. The proof of the convergence of the finite dimensional distributions is a generalization of this proof.

\section{The Matheron And De Marsily Model: RANDOM Vertical ONE-WAY STREets}

We consider a nearest neighbourghs random walk on $\mathbb{Z}^{2}$ with random orientations of the vertical lines. This model has been introduced by Matheron and de Marsily in [31] to model the displacement of a pollutant in a inhomogeneous stratified media, the strata being represented vertically in our representation of this model.

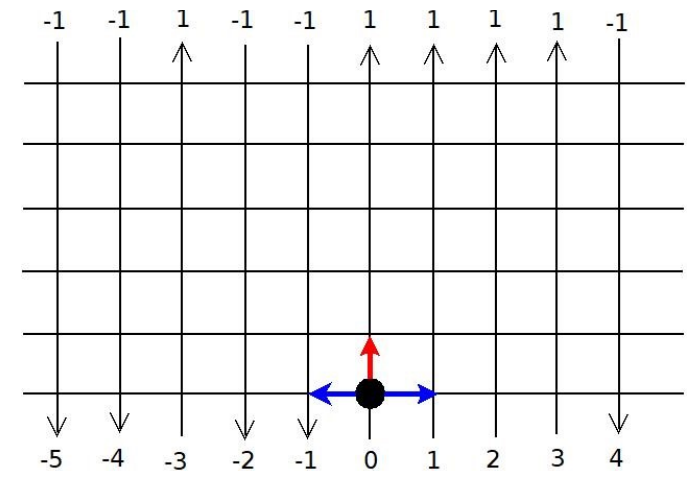

\subsection{Mathematical model}

Mathematically, to every $x \in \mathbb{Z}$, we associate a random variable $\varepsilon_{x}$. The random variables $\varepsilon_{x}$ are assumed to be independent and such that $\mathbb{P}\left(\varepsilon_{x}=1\right)=\mathbb{P}\left(\varepsilon_{x}=-1\right)=\frac{1}{2}$ (centered Rademacher random variables). The vertical line $x$ is then oriented upward if $\varepsilon_{x}=1$ and is oriented downward if $\varepsilon_{x}=-1$. 
The $\left(\varepsilon_{x}\right)_{x}$ being given, we consider a random walker starting from 0 , moving to one of the nearest accessible neighbourghs (with respect to the orientation) with probability $1 / 3$. This means that a walker located at position $(x, y) \in \mathbb{Z}^{2}$ can

- either, with probability $1 / 3$, go left,

- or, with probability $1 / 3$, go right,

- or, with probability $1 / 3$, go vertically with respect to the orientation of the vertical line $x$, which is given by $\varepsilon_{x}$.

Thus the position $M_{n}$ of the walker at time $n$ is given by

$$
M_{n}=\left(S_{n}, \sum_{k=1}^{n} \varepsilon_{S_{k}} \mathbf{1}_{\left\{S_{k}=S_{k-1}\right\}}\right) \text {, }
$$

where $\left(S_{n}\right)_{n}$ is a lazy random walk independent of $\left(\varepsilon_{x}\right)_{x}$ with increments uniformly distributed on $\{-1,0,1\}$.

\subsection{Transience of $\left(M_{n}\right)_{n}$}

In [11], Campanino and Petritis proved the transience of $\left(M_{n}\right)_{n}$, whereas the analogous model with alternate orientations of the vertical lines (i.e. same model but with $\varepsilon_{x}=(-1)^{x}$ ) is recurrent.



Random orientation of vertical lines: The random walk is transient



Alternate orientation of vertical lines: The random walk is recurrent

At a first look, these different behaviours can appear a bit surprising because in both models, in some ways, 50 percent of the vertical lines are oriented upward (resp. downward).

\subsection{Limit theorems}

A key remark in the establishment of limit theorems for $\left(M_{n}\right)_{n}$ is that the second coordinate $M_{n}^{(2)}$ of $M_{n}$ behaves as a random walk in random scenery. Indeed

$$
M_{n}^{(2)}=\sum_{k=1}^{n} \varepsilon_{S_{k}} \mathbf{1}_{\left\{S_{k}=S_{k-1}\right\}} \approx \frac{1}{3} \sum_{k=1}^{n} \varepsilon_{S_{k}} .
$$

This idea can be made rigourous to prove limit theorems.

Distributional limit Theorem. In [22], Le Ny and Guillotin-Plantard proved that

$$
\left(n^{-1 / 2} M_{n t}^{(1)}, n^{-3 / 4} M_{n t}^{(2)}\right)_{t} \stackrel{\mathcal{L}, J_{1}}{\longrightarrow}\left(\mathcal{W}_{t}, \Delta_{t}:=\int_{\mathbb{R}} L_{t}(x) d Y_{x}\right)_{t},
$$

where $Y$ and $\mathcal{W}$ are two independent Brownian motions, and where $\left(L_{t}(x)\right)_{t, x}$ is the local time of $\mathcal{W}$.

Local limit theorem. In [13], Castell, Guillotin-Plantard, Schapira and the author established the following 
local limit theorem

$$
\exists c>0, \quad \mathbb{P}\left(M_{2 n}=(0,0)\right) \sim c n^{-\frac{1}{2}-\frac{3}{4}}=c n^{-\frac{5}{4}} .
$$

Observe that this local limit theorem gives another proof of the transience of $M$ since it directly gives $\sum_{n \geq 1} \mathbb{P}\left(M_{n}=\right.$ $(0,0))<\infty$.

\subsection{Persistence probability in the upper halfplane}

In [1], Aurzada, Guillotin-Plantard and the author answered the question asked by Matheron and de Marsily about persistence and proved that

$$
\mathbb{P}\left(\max _{k=1, \ldots, n} M_{k}^{(2)} \leq-1\right) \sim \frac{3}{4} \mathbb{E}\left[\sup _{[0,1]} \Delta\right] n^{-\frac{1}{4}} .
$$

We detail now the very easy proof of this result, which, as noticed in [1], can be adapted to study the persistence probability for general processes with stationary increments.

Proof of this persistence result. The result is based on the following very simple argument. First, an important remark is that, since the increments of $M^{(2)}$ have length 0 or 1 and since $M^{(2)}$ has the same distribution as $-M^{(2)}$, we have

$$
\mathbb{P}\left(\max _{k=1, \ldots, n} M_{k}^{(2)} \leq-1\right)=\frac{1}{2} \mathbb{P}\left(T_{0}^{(2)}>n\right), \quad \text { with } T_{0}^{(2)}:=\inf \left\{k \geq 1: M_{k}^{(2)}=0\right\} .
$$

Since the increments of $M^{(2)}$ are stationary, it is classical to notice that this quantity is related to the expectation of the range of $M^{(2)}$ in the following manner (by considering the last visit times before time $n$ )

$$
\begin{aligned}
\mathbb{E}\left[\#\left\{M_{k}^{(2)}, k=1, \ldots, n\right\}\right] & =\mathbb{E}\left[\#\left\{k=1, \ldots, n: \forall m=1, \ldots, n-k, M_{k+m}^{(2)}-M_{k}^{(2)} \neq 0\right\}\right] \\
& =\sum_{k=1}^{n} \mathbb{P}\left(T_{0}^{(2)}>n-k\right)=\sum_{k=0}^{n-1} \mathbb{P}\left(T_{0}^{(2)}>k\right) .
\end{aligned}
$$

Classically (see Dvoretzky and Erdös work [18]), this relation is used to go from an estimate of $\mathbb{P}\left(T_{0}^{(2)}>n\right)$ (established thanks to a local limit theorem combined with some independence or mixing property) to an estimate of $\mathbb{E}\left[\#\left\{M_{k}^{(2)}, k=1, \ldots, n\right\}\right]$. Here we use this relation the other way round. Indeed, since the increments of $M^{(2)}$ have length 0 or 1 and since $M^{(2)}$ has the same distribution as $-M^{(2)}$, the expectation of the range of $M^{(2)}$ can be directly estimated as follows

$$
\begin{aligned}
\mathbb{E}\left[\#\left\{M_{k}^{(2)}, k=1, \ldots, n\right\}\right] & =\mathbb{E}\left[\max _{k=1, \ldots, n} M_{k}^{(2)}-\min _{k=1, \ldots, n} M_{k}^{(2)}+1\right] \\
& =2 \mathbb{E}\left[\max _{k=1, \ldots, n} M_{k}^{(2)}\right]+1 .
\end{aligned}
$$

Therefore

$$
2 \sum_{k=1}^{n} \mathbb{P}\left(\max _{j=1, \ldots, k} M_{j}^{(2)} \leq-1\right)=\sum_{k=0}^{n-1} \mathbb{P}\left(T_{0}^{(2)}>k\right)=2 \mathbb{E}\left[\max _{k=1, \ldots, n} M_{k}^{(2)}\right]+1 \sim 2 n^{\frac{3}{4}} \mathbb{E}\left[\sup _{[0,1]} \Delta\right],
$$

where we used the convergence in distribution of $\left(\left(M_{\lfloor n t\rfloor} / n^{3 / 4}\right)_{t}\right)_{n}$ combined with some uniform integrability argument. We conclude that $\mathbb{P}\left(\max _{j=1, \ldots, n} M_{j}^{(2)} \leq-1\right) \sim \frac{3}{4} n^{-\frac{1}{4}} \mathbb{E}\left[\sup _{[0,1]} \Delta\right]$, since this probability is decreasing 
in $n$.

\subsection{Open model: Random Manhattan model}

Now we consider a nearest neighbourghs random walk $\widetilde{M}$ in $\mathbb{Z}^{2}$ with, this time, random orientations of both the horizontal and vertical lines.

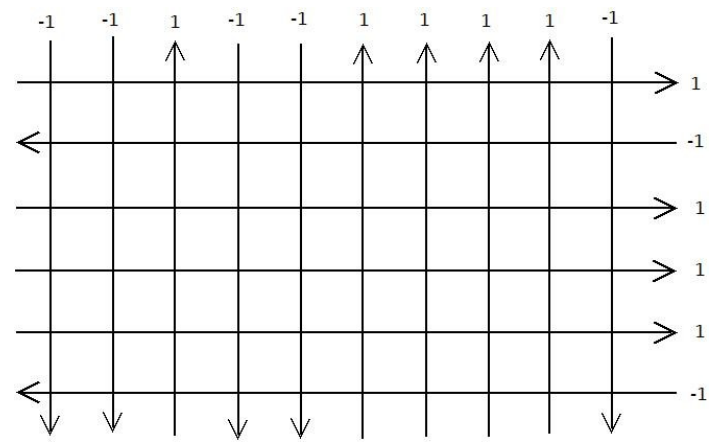

We consider two independent families $\left(\varepsilon_{x}^{(1)}\right)_{x \in \mathbb{Z}}$ and $\left(\varepsilon_{x}^{(2)}\right)_{x \in \mathbb{Z}}$ of independent centered Rademacher random variables. The vertical line $x$ is oriented upward if $\varepsilon_{x}^{(2)}=1$ and is oriented downward if $\varepsilon_{x}^{(2)}=-1$. The $y$-th horizontal line is oriented rightward if $\varepsilon_{y}^{(1)}=1$ and is oriented leftward if $\varepsilon_{y}^{(1)}=-1$.

The $\varepsilon=\left(\varepsilon_{x}^{(1)}, \varepsilon_{x}^{(2)}\right)_{x \in \mathbb{Z}}$ being given, we consider a random walk $\widetilde{M}$ starting from $(0,0)$ and moving randomly to one of its nearest neighbouring sites with respect to the orientations of the lines.

In this model, a walker located at position $(x, y) \in \mathbb{Z}^{2}$ can

- either, with probability $1 / 2$, make one step in the vertical direction with respect to the orientation of the vertical line $x$, which is given by $\varepsilon_{x}^{(2)}$,

- or, with probability $1 / 2$, make one step in the horizontal direction with respect to the orientation of the horizontal line $y$, which is given by $\varepsilon_{y}^{(1)}$.

Formally, given $\varepsilon, \widetilde{M}$ is a Markov chain such that

$$
\widetilde{M}_{0}=(0,0) \quad \text { and } \quad P^{\varepsilon}\left(\widetilde{M}_{n+1}=\left(x+\varepsilon_{y}^{(1)}, y\right) \mid \widetilde{M}_{n}=(x, y)\right)=P^{\varepsilon}\left(\widetilde{M}_{n+1}=\left(x, y+\varepsilon_{x}^{(2)}\right) \mid \widetilde{M}_{n}=(x, y)\right)=\frac{1}{2}
$$

This model is the random version of the so-called Manhattan model in which the orientations are alternate $\varepsilon_{x}^{(1)}=\varepsilon_{x}^{(2)}=(-1)^{x}$ and which behaves as a simple random walk in $\mathbb{Z}^{2}$ as proved in [32]. 

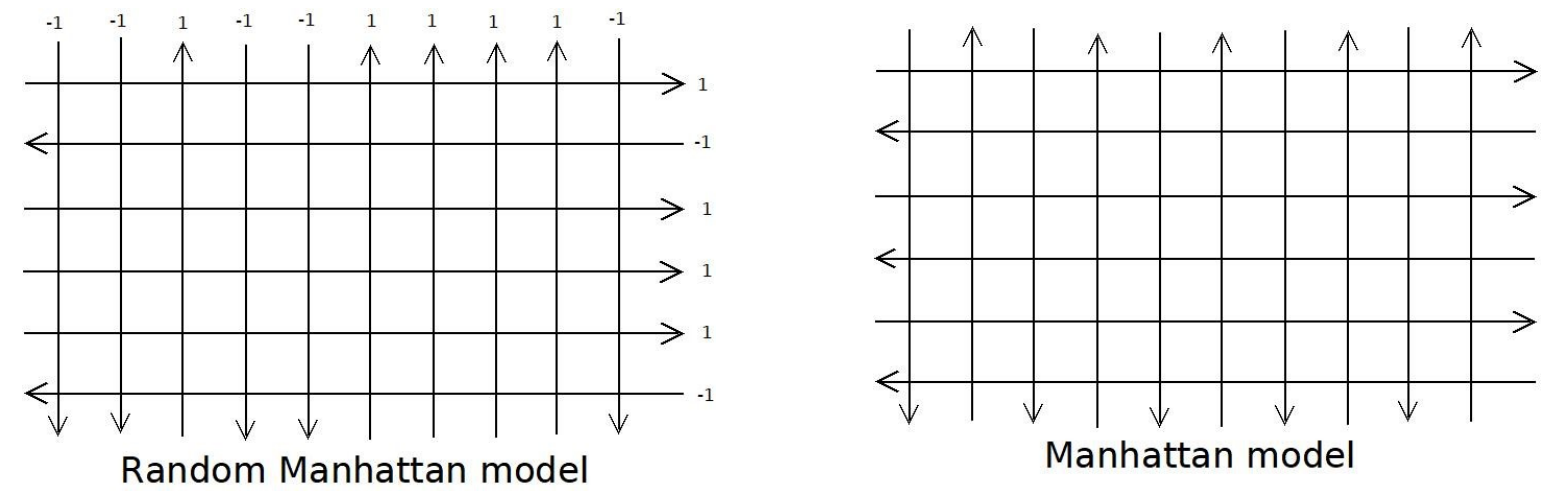

More precisely, for the classical Manhattan model $\left(M_{n}^{0}\right)_{n}$, Guillotin-Plantard observed that $\left(S_{n}:=\psi\left(M_{2 n}^{0}\right)\right)_{n}$ is a simple symmetric random walk on $\mathbb{Z}^{2}$ where $\psi:(x, y) \mapsto(\lfloor x / 2\rfloor,\lfloor y / 2\rfloor)$ and that $S_{2 n}=\psi\left(M_{4 n}^{0}\right)=(0,0) \Leftrightarrow$ $M_{4 n} \in\{(0,0),(1,1)\}$, so $\mathbb{P}\left(S_{2 n}=(0,0)\right)=\mathbb{P}\left(M_{4 n}^{0}=(0,0)\right)+\mathbb{P}\left(M_{4 n}^{0}=(1,1)\right)=2 \mathbb{P}\left(M_{4 n}^{0}=(0,0)\right)$ for symmetry reasons.

Let's come back to the random Manhattan model. As for the previous model $M$, we can express the second coordinate of the random Manhattan walk $\widetilde{M}$ using the first coordinate, but the converse is also true and this complicates seriously the study of this model. Indeed $\widetilde{M}_{n}$ can be rewritten as follows

$$
\widetilde{M}_{n}=\left(\sum_{k=1}^{n} \varepsilon_{\widetilde{M}_{k}^{(2)}}^{(1)} \mathbf{1}_{\left\{\widetilde{M}_{k}^{(2)}=\widetilde{M}_{k-1}^{(2)}\right\}}, \sum_{k=1}^{n} \varepsilon_{\widetilde{M}_{k}^{(1)}}^{(2)} \mathbf{1}_{\left\{\widetilde{M}_{k}^{(1)}=\widetilde{M}_{k-1}^{(1)}\right\}}\right) .
$$

Conjecture. We conjecture that $\widetilde{M}_{n}$ has order $n^{2 / 3}$.

A rough argument leading to this conjecture. Assume $M_{n}^{(1)} \approx \alpha_{n}$ and so $M_{n}^{(2)} \approx \alpha_{n}$ (for symmetry reason). We should have $M_{n}^{(1)} \approx \sum_{y} \varepsilon_{y}^{(1)} N_{n}^{(2)}(y)$ with $N_{n}^{(2)}(y)$ the local time of $\widetilde{M}_{n}^{(2)}$. It is reasonnable to think that $N_{n}^{(2)}(y) \approx \frac{n}{\alpha_{n}}$ for most of the $y$ such that $N_{n}^{(2)}(y)>0$, this leads to $\alpha_{n} \approx M_{n}^{(1)} \approx \sum_{y} \varepsilon_{y}^{(1)} N_{n}^{(2)}(y) \approx\left(\alpha_{n}\right)^{1 / 2} \frac{n}{\alpha_{n}}$ and so to $\alpha_{n} \approx n^{2 / 3}$.

A first step has been done in this direction by Ledger, Tóth and Valkó in [29] who proved, for the analogous continuous-time model $\mathcal{M}_{t}$, the following estimate

$$
\exists C>0, \quad \exists \lambda_{0}>0, \quad \forall \lambda \in\left(0, \lambda_{0}\right), \quad C^{-1} \lambda^{-\frac{9}{4}} \leq \int_{0}^{+\infty} e^{-\lambda t} \mathbb{E}\left[\left|\mathcal{M}_{t}\right|^{2}\right] d t \leq C \lambda^{-\frac{5}{2}},
$$

which is close to say that $c t^{\frac{5}{4}} \leq \mathbb{E}\left[\left|\mathcal{M}_{t}\right|^{2}\right] \leq c^{\prime} t^{\frac{3}{2}}$ and so close to say that $c_{0} n^{\frac{5}{8}} \leq\left\|\widetilde{M}_{n}\right\|_{2} \leq c_{0}^{\prime} n^{\frac{3}{4}}$ (the link between $\mathcal{M}_{t}$ and $\widetilde{M}_{n}$ is that $\widetilde{M}_{n}$ has the same distribution as $\mathcal{M}_{\tau_{n}}$, where $\tau_{n}$ is the $n$-th jump time of $\mathcal{M}$ which is a sum of independent identically distributed exponential random variables, therefore $\tau_{n} \sim n \mathbb{E}\left[\tau_{1}\right]$ almost surely).

\section{ONE-DIMENSIONAL LÉVY-LORENTZ GAS: RANDOM ROUNDABOUTS ON THE LINE}

We present now a last model: the model of one-dimensional Lévy-Lorentz gas that was introduced in [2] as a one-dimensional toy model for transport in porous media.

\subsection{Description of the model}

On $\mathbb{R}$, we put roundabouts at positions $R:=(R(k))_{k \in \mathbb{Z}}$ in such a way that $R(0)=0$ and that the gaps between two consecutive roundabouts are given by independent identically distributed positive random variables 
$\zeta_{k}:=R(k)-R(k-1)$

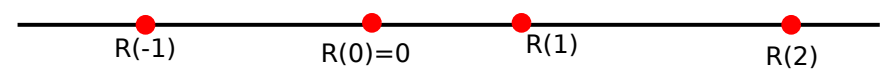

Continuous time process $X$ : Knowing $R$, we consider a process $\left(X_{t}\right)_{t}$ that

- starts from 0 , by going either to the right or to the left (both with probability $1 / 2$ ),

- moves at unit speed, goes straight between consecutive roundabouts,

- goes straight or turns back (with probability $1 / 2$ ) at each roundabout,

all these choices being made independently.

This model has been introduced by Barkai, Fleurov and Klafter in [2].

\subsection{A quenched central limit theorem in the integrable case}

Bianchi, Cristadoro, Lenci and Ligabò studied in [4] the case $\mathbb{E}[R(1)]<\infty$ and proved that

$$
\text { for a.e. } R, \quad t^{-\frac{1}{2}} X_{t} \stackrel{\mathcal{L}}{\longrightarrow} \sqrt{\mathbb{E}[R(1)]} Z \text { as } t \rightarrow+\infty \text {, }
$$

with $Z$ a standard Gaussian random variable.

They also conjectured that the normalization is not always the same for the annealed variance of $X_{t}$ and that $\mathbb{E}\left[\left(X_{t}\right)^{2}\right] \approx t^{\max \left(1, \frac{5}{2}-\beta\right)}$ if $\mathbb{P}\left(\zeta_{1}>z\right) \approx z^{-\beta}$ as $z \rightarrow+\infty$, for some $\beta>1$.

\subsection{A Limit theorem in an non-integrable case}

Bianchi, Lenci and the author studied in [5] the case when the distribution of $R(1)$ is in the normal domain of attraction of a $\beta$-stable distribution, i.e. when $n^{-\frac{1}{\beta}} R(n) \stackrel{\mathcal{L}}{\longrightarrow} Y_{1}$, with $0<\beta<1$ (so that $\mathbb{E}[R(1)]=\infty$ ). Under this assumption, we proved that

$$
\left(n^{-\frac{1}{\beta+1}} X_{n t}\right)_{t} \stackrel{\mathcal{L}, \text { f.d.d. }}{\longrightarrow}\left(\mathcal{X}_{t}\right)_{t}, \text { as } n \rightarrow+\infty
$$

where $\mathcal{X}$ is a non càdlàg process.

Scheme of the proof. The key idea is that $\left(X_{t}\right)_{t}$ can be modeled by a simple symmetric random walk $S$ on $\mathbb{Z}$, up to a change of time (which is given by a RWRS with the random walk $S$ ) and up to a change of space (which is given by the scenery).

Recall that $\zeta_{k}=R(k)-R(k-1)$ are independent and identically distributed positive random variables satisfying $n^{-\frac{1}{\beta}} R(n) \stackrel{\mathcal{L}}{\longrightarrow} Y_{1}$, with $0<\beta<1$.

We set $S(n)$ for the index of the $n$-th roundabout met by the process $X$ and we set $\tau(n)$ for the time at which the process $X$ reaches this roundabout. Observe that $\left(S_{n}\right)_{n}$ is a simple symmetric random walk on $\mathbb{Z}$ and that

$$
X_{\tau(n)}=R(S(n))
$$

and so that, in some sense,

$$
X_{t} \approx R\left(S\left(\tau^{-1}(t)\right)\right), \quad \text { with } \tau^{-1}(s):=\sup \left\{u>0: \tau_{\lfloor u\rfloor}<s\right\}
$$

We know that

$$
\left(N^{-\frac{1}{\beta}} R(\lfloor N t\rfloor)\right)_{t \in \mathbb{R}} \stackrel{\mathcal{L}}{\longrightarrow}\left(Y_{t}\right)_{t \in \mathbb{R}} \quad \text { and } \quad\left(m^{-\frac{1}{2}} S(\lfloor m t\rfloor)\right)_{t} \stackrel{\mathcal{L}}{\longrightarrow}\left(B_{t}\right)_{t}
$$


where $Y=\left(Y_{t}\right)_{t}$ is a $\beta$-stable process and where $B=\left(B_{t}\right)_{t}$ is a standard Brownian motion. Moreover we prove that $\tau_{n}=\sum_{k=1}^{n} \zeta_{\max (S(k-1), S(k))} \approx \sum_{k=1}^{n} \zeta_{S(k)}$, which is a RWRS, from which we conclude that $\left(n^{-1} \tau_{\left\lfloor n^{\frac{2 \beta}{\beta+1}} t\right\rfloor}\right)_{t} \rightarrow\left(\Delta_{t}=\right.$ $\left.\int_{\mathbb{R}} L_{t}(x) d Y(x)\right)_{t}$, with $\left(L_{t}(x)\right)_{t, x}$ the local time of $B$. This leads to

$$
n^{-\frac{2 \beta}{\beta+1}} \tau^{-1}(n t) \rightarrow \Delta^{-1}(t) \text {. }
$$

We prove that the three above convergences hold together and, combining (2), (3) and (4), we conclude that

$$
\left(n^{-\frac{1}{\beta+1}} X_{n t} \approx n^{-\frac{1}{\beta+1}} R\left(n^{\frac{\beta}{\beta+1}} n^{-\frac{\beta}{\beta+1}} S\left(n^{\frac{2 \beta}{\beta+1}} n^{-\frac{2 \beta}{\beta+1}} \tau^{-1}(n t)\right)\right)\right)_{t} \stackrel{\mathcal{L}, \text { f.d.d. }}{\longrightarrow}\left(\mathcal{X}_{t}:=Y_{B_{\Delta^{-1}(t)}}\right)_{t} .
$$

The fact that $\mathcal{X}: t \mapsto Y_{B_{\Delta^{-1}(t)}}$ is not almost surely equal to càdlàg process comes from the fact that $Y$ is not continuous and independent of $B$. So, for every discontinuity point $t_{0}$ of $Y$, a.s., $B$ oscillates around $B\left(t_{0}\right)$ on every interval $] t_{0}-\eta, t_{0}[$ or $] t_{0}, t_{0}+\eta[$.

\section{REFERENCES}

[1] F. Aurzada, N. Guillotin-Plantard, F. Pène, Persistence probabilities for stationary increment processes, Stochastic Processes and Applications 128 (2018), no. 5, 1750-1771.

[2] E. Barkai, V. Fleurov, J. Klafter, One-dimensional stochastic Lévy-Lorentz gas, Phys. Rev. E 61 (2000), no. 2, 1164-1169.

[3] G. Ben Arous, J. Cerný, Scaling limit for trap models on $\mathbb{Z}^{d}$, Ann. Probab. 35 (2007), no. 6, $2356-2384$.

[4] A. Bianchi, G. Cristadoro, M. Lenci, M. Ligabò, Random walks in a one-dimensional Lévy random environment $J$. Stat. Phys. 163 (2016), no. 1, 22-40.

[5] A. Bianchi, M. Lenci, F. Pène, Continuous-time random walk between Lévy-spaced targets in the real line, arXiv:1806.02278.

[6] P. Billingsley, Convergence of probability measure, Second edition. John Wiley \& Sons, Inc., New York (1999).

[7] E. Bolthausen, A central limit theorem for two-dimensional random walks in random sceneries, Annals of Probability $\mathbf{1 7}$ (1989) 108-115.

[8] A. N. Borodin, A limit theorem for sums of independent random variables defined on a recurrent random walk, (Russian) Dokl. Akad. Nauk SSSR 246 (1979), no. 4, 786-787.

[9] A. N. Borodin, Limit theorems for sums of independent random variables defined on a transient random walk, Investigations in the theory of probability distributions, IV. Zap. Nauchn. Sem. Leningrad. Otdel. Mat. Inst. Steklov. (LOMI) 85 (1979), 17-29, 237, 244.

[10] P. Cabus, N. Guillotin-Plantard, Functional limit theorems for U-statistics indexed by a random walk. Stochastic Processes and their Applications 101 (2002) 143-160.

[11] M. Campanino, D. Petritis, Random walks on randomly oriented lattices, Mark. Proc. Relat. Fields 9 (2003), $391-412$.

[12] F. Castell, N. Guillotin-Plantard, F. PÈne, Limit theorems for one and two-dimensional random walks in random scenery. Ann. Inst. H. Poincaré 49 (2013) 506-528.

[13] F. Castell, N. Guillotin-Plantard, F. Pène, Br. Schapira, A local limit theorem for random walks in random scenery and on randomly oriented lattices. Annals of Probability 39 (2011) 2079-2118.

[14] Fabienne Castell, Nadine Guillotin-Plantard, F. Pène, Br. Schapira, On the local time of random processes in random scenery, Annals of Probability 42 (2014), No. 6, 2417-2453

[15] J. Cerny, Moments and distribution of the local time of a two-dimensional random walk. Stochastic Process. Appl. 117 (2007), no. 2, 262-270.

[16] A. Dabrowski, H. Dehling, T. Мıкоsch, O.Sh. Sharipov, Poisson limits for U-statistics, Stochastic Processes and their Applications 99 (2002) 137-157.

[17] G. Deligiannidis, S. Utev, An asymptotic variance of the self-intersections of random walks, Sib. Math. J. 52 (2011) $639-650$.

[18] A. Dvoretzky and P. Erdös, Some problems on random walk in space, Proc. Berkeley Sympos. math. Statist. Probab. (1951), 353-367.

[19] L. R. G. Fontes, P. Mathieu, On the dynamics of trap models in $Z^{d}$. Proceedings of the London Mathematical Society, vol. 6, No 108, 1562-1592, 2014.

[20] B. Franke, F. Pène, M. Wendler, Convergence of U-statistics indexed by a random walk to stochastic integrals of a Levy sheet, Communications in Algebra 45 (2017), No 2 (2017), 606-620. 
[21] B. Franke, F. PÈne, M. Wendler, Stable Limit Theorem for U-Statistic Processes Indexed by a Random Walk, Electronic Communications in Probability 22 (2017), No. 9, 1-12.

[22] N. Guillotin-Plantard, A. Le Ny. A functional limit theorem for a 2d-random walk with dependent marginals. Electronic Communications in Probability, 13 (2008), 34, 337-351.

[23] N. Guillotin-Plantard, V. Ladret, Limit theorems for U-statistics indexed by a one dimensional random walk, ESAIM 9 (2005) 95-115.

[24] L. Heinrich, W. Wolf, On the convergence of U-statistics with stable limit distribution, Journal of Multivariate Analysis 44 (1993) 266-278.

[25] W. Hoeffing, A class of statistics with asymptotically normal distribution, Ann. Math. Stat. 19, (1948), 293-325. MR 0026294

[26] S. A. Kalikow, T, $T^{-1}$ Transformation is Not Loosely Bernoulli, Annals of Mathematics, Second Series, 115 (1982), No. 2, 393-409.

[27] H. Kesten, F. Spitzer, A limit theorem related to an new class of self similar processes, Zeitschrift für Wahrscheinlichkeitstheorie und verwandte Gebiete 50 (1979) 5-25.

[28] J.-F. Le Gall, J. Rosen, The range of stable random walks., Ann. Probab. 19 (1991), 650-705.

[29] S. Ledger, B. Tóth, B. Valkó, Random walk on the randomly-oriented Manhattan lattice, Electron. Commun. Probab. 23 (2018), paper no. 43, 11 pp.

[30] S. Lounichi, E. Rio, Functional convergence to Lévy motions for iterated random Lipschitz mappings, Electron. J. Probab. 16 (2011) 2452-2480.

[31] G. Matheron, G. De Marsily, Is transport in porous media always diffusive? A counterxample, Water Resources Res., 16 (1980) 901-907.

[32] N. Guillotin, Marche aléatoire dynamique dans une scène aléatoire. Problèmes liés à l'inhomogénéité spatiale de certaines chaînes de Markov, PhD thesis, Université de Rennes 1 (1997).

[33] K. Schmidt, On recurrence, Z. Wahrsch. Verw. Gebiete, 68 (1984), 75-95.

[34] K. Schmidt, Recurrence of cocycles and stationary random walks, IMS Lecture Notes-Monograph Series, Dynamics \& Stochastics, 48 (2006) 78-84.

[35] F. Spitzer, Principles of random walks, Van Nostrand, Princeton, N.J. (1964).

[36] B. Weiss, The isomorphism problem in ergodic theory, Bull. A.M.S. 78 (1972), 668-684.

[37] W. Wнiтt, Stochastic-process limits. An introduction to stochastic-process limits and their application to queues, Springer Series in Operations Research. Springer-Verlag, New York (2002). 